\title{
Caracterización fisicoquímica y nutricional del fruto Butia capitata y de su pulpa tamizada
}

\author{
Crosa, M. J. (1)*, Burzaco, P. (1), Pastorino, N. (1), Irisity, M. (2), Gioscia, D. (1), Ayres, C. (2) \\ (1) Gerencia Proyectos Alimentarios. Laboratorio Tecnológico del Uruguay, LATU - (2) Departamento Gestión y Trans- \\ ferencia Tecnológica para el Desarrollo Local, Gerencia de Gestión Empresarial. Laboratorio Tecnológico del Uruguay, \\ LATU.
}

Contacto: caayres@latu.org.uy

Recibido: 28/06/2011 - Aprobado: 8/12/2011

\begin{abstract}
Resumen
El butiá es un fruto silvestre proveniente de los palmares de Rocha, cuyo consumo y comercialización se limita principalmente a ese departamento. El análisis de las propiedades fisicoquímicas y nutricionales en el fruto fresco y en su pulpa tamizada favorecerá la valorización de un recurso genético nativo. El objetivo de este estudio fue ampliar el conocimiento acerca de las características físico químicas y nutricionales del fruto fresco y de su pulpa tamizada, y estudiar la evolución microbiológica de la pulpa tamizada almacenada a $-18{ }^{\circ} \mathrm{C}$ durante 360 días. La caracterización del fruto fresco se realizó mediante los ensayos de sólidos solubles totales, pH, color, azúcares reductores, acidez titulable, materia seca, pectina, fibra alimentaria total y vitamina $\mathrm{C}$. Los resultados más relevantes revelaron un contenido de vitamina $\mathrm{C}$ del entorno del 65 a 100 ppm de vitamina $C$ y un contenido de fibra alimentaria de 4,9\% a 4,3\%. En la pulpa tamizada se analizó sólidos solubles totales, $\mathrm{pH}$, color y vitamina $\mathrm{C}$ y pectina, microorganismos mesófilos aerobios, hongos y levaduras. El principal resultado fue la preservación de hasta un $55 \%$ del contenido de vitamina $\mathrm{C}$ en la pulpa tamizada con respecto al fruto fresco. Durante los 360 días de almacenamiento a $-18{ }^{\circ} \mathrm{C}$ no se observó crecimiento microbiano en la pulpa tamizada.

Palabras clave: Butia capitata, butiá, pulpa tamizada, propiedades químicas y nutricionales, fruto nativo, almacenamiento pulpa tamizada.
\end{abstract}

\begin{abstract}
$\underline{\text { Abstract }}$
Butiá is a native fruit from the Butiá Palms in the east of Uruguay, whose consumption and commercialization is limited to this region. The analysis of physicochemical and nutritional properties in the fruit and its screened pulp will encourage the enhancement of this native genetic resource. The aim of this study was to expand knowledge about the physical, chemical and nutritional characteristics of the fresh fruit and its screened pulp. We also studied the microbiological evolution of the sived pulp stored at $-18^{\circ} \mathrm{C}$ for 360 days. In order to characterize the fruit, measurements of total soluble solids, $\mathrm{pH}$, reducing sugars, titrable acidity, dry matter, pectin, total dietary fiber and vitamin $\mathrm{C}$ were carried out. Most relevant results obtained from the fruit were 65 to $100 \mathrm{ppm}$ of vitamin $\mathrm{C}$ and 4,3 to $4,9 \%$ of total dietary fiber. In the screened pulp, total soluble solids, pH, color, vitamin $\mathrm{C}$, pectin, aerobic mesophilic microorganisms, fungi and yeast were analyzed. The high content of vitamin $\mathrm{C}$ in the fruit was preserved up to $55 \%$ in the screened pulp. During the 360 days of storage at $-18^{\circ} \mathrm{C}$ no microbiological growth in screened pulp were found. Keywords: Butia capitata, butiá, sieved butiá pulp, chemical and nutritional properties, native fruit, sieved butiá pulp preservation.
\end{abstract}

\section{Introducción}

Este estudio fue financiado por el Fondo de Promoción de Tecnología Agropecuaria del Instituto Nacional de Investigación Agropecuaria (INIA), en el marco del proyecto "Aprovechamiento agroalimentario del fruto de la palmera butiá (Butia capitata) FPTA 178", cuyo principal objetivo es promover el desarrollo local sostenible en la zona del Este de Uruguay mediante la innovación y mejora de productos vinculados al fruto del Butiá. El equipo de proyecto se caracteriza por su multidisciplinariedad de enfoques y trabajo interinstitucional.

La especie Butia capitata de la familia Arecaceae (Palmae) es una de las palmeras más australes del mundo. Se distribuye en el sur de
Brasil, en los estados de Santa Catarina y Rio Grande do Sul, y en el este de Uruguay, en los departamentos de Cerro Largo, Treinta y Tres, Lavalleja, Maldonado y Rocha. Las poblaciones actuales de la especie están formadas por individuos coetáneos y centenarios, debido a la ausencia de regeneración de estos palmares (Rivas y Barilani, 2004). Conjuntamente con la conservación de la diversidad biológica y de un paisaje único, la caracterización del fruto fresco y su aprovechamiento en productos elaborados por pobladores locales forman parte de la estrategia para el desarrollo local sostenible.

Se desarrollaron equipamientos para el aprovechamiento de la almendra del fruto, la fibra insoluble y la pulpa tamizada (Betancurt et al., 2008). En la actualidad existen en el departamento de Rocha no menos de 40 emprendimientos que cosechan el fruto y lo almacenan congelado con el objetivo de consumirlo durante el año. La pulpa 
tamizada del Butiá es el principal componente para el desarrollo de los productos y, por lo tanto, el que requiere mayor atención. En el período 2010-2011 se han desarrollado cartillas de elaboración de productos en base a este fruto que fueron difundidas y transferidas a elaboradores de la zona (Burzaco et al., 2010a; 2010b; 2010c).

El objetivo de este trabajo fue ampliar el conocimiento acerca de las características físico químicas y nutricionales del fruto fresco y de su pulpa tamizada, así como estudiar la evolución microbiológica de la pulpa tamizada almacenada por congelación durante 360 días.

\section{Materiales y Métodos}

\section{Descripción de cosecha y almacenamiento del fruto}

Las muestras de fruto fresco se obtuvieron en un predio agropecuario ubicado en un palmar en la ruta 16 (Camino del Indio) a $5 \mathrm{~km}$ al norte de la ciudad de Castillos durante la zafra 2010. Los frutos se recolectaron según el procedimiento tradicional de cosecha: desprendimiento del cacho en forma mecánica y, una vez en el piso, selección de frutos de acuerdo a su integridad, firmeza y color. A partir de un muestreo aleatorio se extrajeron 5 muestras de $1 \mathrm{~kg}$ para analizar las propiedades fisicoquímicas del fruto fresco. Del resto de la cosecha se seleccionaron frutos en el tono de amarillo y naranja, por ser los que se presentan con mayor frecuencia en esta región, y se dividieron en 4 lotes de $60 \mathrm{~kg}$ para los ensayos de elaboración de pulpa en escala piloto.

Inmediatamente luego de cosechadas, las muestras destinadas para análisis del fruto fresco se refrigeraron a $5{ }^{\circ} \mathrm{C}$ durante $48 \mathrm{hrs}$, se desinfectaron y se conservaron por un máximo de un mes en cámara de $-18{ }^{\circ} \mathrm{C}$. Antes de ser analizadas se descongelaron dentro de las bolsas hasta lograr temperatura ambiente. Para el ensayo de elaboración de pulpa tamizada las muestras recién cosechadas se mantuvieron refrigeradas a $5{ }^{\circ} \mathrm{C}$ durante $48 \mathrm{~h}$ y al tercer día se elaboró la pulpa tamizada.

\section{Descripción del proceso de elaboración de la pulpa tamizada}

Para la elaboración de la pulpa tamizada se procesaron 4 lotes de $60 \mathrm{~kg}$ de fruta. Luego del lavado y desinfección se procedió a la cocción del fruto en paila con camisa con vapor a $1,5 \mathrm{~kg} / \mathrm{cm}^{2} \mathrm{de}$ presión. Antes de iniciar el calentamiento se agregó $24 \mathrm{~kg}$ de agua, se llevó a ebullición durante 30 minutos y posteriormente se realizaron 3 pasajes por tamiz de $3 \mathrm{~mm}$. Se realizó un muestreo por duplicado de cada lote de producción para su análisis.

La pulpa elaborada se envasó en bolsas de polietileno en porciones de $2 \mathrm{~kg}$ y se almacenó en cámara a $-18^{\circ} \mathrm{C}$.

Se realizó toma de muestra en la pulpa tamizada congelada en los días $0,90,180,270$ y 360 . En cada día de muestreo se seleccionaron aleatoriamente 2 muestras para los ensayos microbiológicos y 4 muestras para la medida de color.

\section{Tratamiento estadístico de los datos}

Los resultados de análisis se presentan en forma de tablas, detallando el intervalo de confianza de los valores con un nivel de significancia del $95 \%$. Los valores informados corresponden al promedio del duplicado de cada ensayo.

\section{Descripción de procedimientos analíticos}

\section{Fruto fresco}

Se realizaron las siguientes medidas de caracterización del fruto fresco: sólidos solubles totales (SST), con refractómetro de mano ATAGO N-1E (0-32\%Brix); pH, con pHmetro portátil Jenway 350; azúcares reductores, mediante titulación automática, aplicando el Compendium of International Methods of Analisys (OIV A4); acidez titulable (AT), expresada en ácido cítrico por valoración potenciométrica basada en la técnica ISO 750 humedad, por método gravimétrico a presión reducida, según Kirk et al. (1991); vitamina $\mathrm{C}$, por cromatografía líquida, según Interlaboratorio (Brause et al., 2003) pectina, mediante método enzimático espectrofotométrico con kit Megazyme para determinación de pectina en alimentos (KPECID); fibra alimentaria total, por método enzimático gravimétrico, según AOAC, 1996- método 985.29. Se calculó la acidez en base seca y la relación entre los sólidos solubles totales y la acidez titulable (SST/ AT o ratio).

Se analizó además el peso de pulpa, cáscara y coco de 40 unidades de fruto tomados aleatoriamente de las muestras cosechadas, y el Color Pantone, por comparación de escala Pantone Formula Guide/ Solid coated en muestras de pulpa del fruto fresco.

\section{Pulpa tamizada}

Se realizaron las siguientes medidas de caracterización de la pulpa tamizada: sólidos solubles totales, con refractómetro de mano ATAGO $\mathrm{N}-1 \mathrm{E}$ (0-32\%Brix); $\mathrm{pH}$, con pHmetro portátil Jenway 350; vitamina $\mathrm{C}$, por cromatografía líquida, según Interlaboratorio (Brause et al., 2003); pectina, mediante método enzimático espectrofotométrico con kit Megazyme para determinación de pectina en alimentos (KPECID), y Color Pantone, por comparación de escala Pantone Formula Guide/ Solid coated.

El cálculo de rendimiento en la producción de pulpa tamizada se realizó por medio del control del peso inicial del fruto fresco y el peso final de pulpa obtenida.

\section{Pulpa tamizada congelada}

Para el seguimiento microbiológico de la pulpa tamizada congelada se aplicaron las siguientes técnicas de análisis: recuento de Hongos y Levaduras, según APHA, 2001a y recuento de aerobios totales, basado en APHA, 2001b y APHA, 2001c.

\section{Resultados}

\section{Caracterización del butiá fresco}

En la Tabla 1 se presentan los resultados de los análisis realizados en las muestras recolectadas del fruto fresco.

En la Tabla 2 se presenta el peso promedio de cada uno de los componentes del fruto fresco.

\section{Caracterización de la pulpa tamizada y rendimiento del proceso de elaboración}

En la Tabla 3 se expresan los resultados de análisis de cada lote de pulpa tamizada producida en escala piloto.

En la Tabla 4 se describen los colores observados en las muestras de fruto fresco y de pulpa tamizada.

El rendimiento del proceso de producción de pulpa tamizada fue del $(50 \pm 1) \%$ en los cuatro lotes estudiados. 


\begin{tabular}{|c|c|c|c|c|c|c|c|c|c|c|}
\hline & \multicolumn{10}{|c|}{ FRUTO FRESCO - ZAFRA 2010} \\
\hline & SST $\left({ }^{\circ} \mathbf{B x}\right)$ & PH & $\begin{array}{c}\text { Azúcares } \\
\text { Reductores (g/l) } \\
\end{array}$ & \begin{tabular}{|c|} 
Acidez titulable \\
(AT) $(\mathrm{g} / \mathbf{1 0 0 g r})$
\end{tabular} & $\begin{array}{c}\text { Acidez (g/100gr } \\
\text { sólido seco) }\end{array}$ & $\begin{array}{c}\text { Humedad } \\
\text { (g/100gr pulpa) }\end{array}$ & $\begin{array}{c}\text { Vitamina } \mathrm{C} \\
\text { (mg/100gr pulpa) }\end{array}$ & $\begin{array}{l}\text { Pectina } \\
(\mathrm{g} / \mathrm{mol})\end{array}$ & \begin{tabular}{|c|} 
Fibra Alimentaria \\
Total (g/100gr pulpa)
\end{tabular} & SST/AT \\
\hline MUESTRA 1 & 16 & 3,1 & 42 & 1,3 & 6,81 & 81 & 66 & $<\mathrm{LD}$ & 4,7 & 12 \\
\hline MUESTRA 2 & 15 & 3,2 & 44 & 1,6 & 8,89 & 82 & 96 & $<\mathrm{LD}$ & 4,7 & 9 \\
\hline MUESTRA 3 & 15 & 3,1 & 45 & 1,6 & 8,70 & 82 & 74 & $<\mathrm{LD}$ & 4,5 & 9 \\
\hline MUESTRA 4 & 16 & 3,0 & 54 & 1,5 & 8,67 & 83 & 90 & $<\mathrm{LD}$ & SD & 10 \\
\hline MUESTRA 5 & 15 & 3,1 & 38 & 1,6 & 8,04 & 80 & 88 & $<\mathrm{LD}$ & SD & 9 \\
\hline PROMEDIO & 15 & 3,1 & 45 & 1,5 & 8 & 81 & 83 & $<L D$ & 4,6 & 10 \\
\hline IC95\% & 0,5 & 0,10 & 8 & 0,2 & 1 & 1 & 17 & - & 0,3 & 2 \\
\hline Límite superior, IC95\% & 15,5 & 3,2 & 53 & 1,7 & 9 & 83 & 100 & $<L D$ & 4,9 & 12 \\
\hline Límite inferior, IC95\% & 14,5 & 3,0 & 36 & 1,3 & 7 & 80 & 65 & $<L D$ & 4,3 & 8 \\
\hline
\end{tabular}

Tabla 1. Propiedades fisicoquímicas de butiá fresco, zafra 2010. Ensayos realizados en la pulpa (L.D. = Límite de detección, $8 \mathrm{E}-9 \mathrm{~g} / \mathrm{mol} ; \mathrm{SD}=$ sin datos).

\begin{tabular}{|l|c|}
\hline Componentes & Peso (gramos) \\
\hline Fruto & $9,3 \pm 0,7$ \\
\hline Cáscara & $0,97 \pm 0,1$ \\
\hline Pulpa & $6,0 \pm 0,6$ \\
\hline Endocarpo leñoso (Coco) & $2,3 \pm 0,2$ \\
\hline
\end{tabular}

Tabla 2. Composición en gramos de cáscara, pulpa y endocarpo leñoso (coco) del fruto fresco, zafra 2010.

\begin{tabular}{|c|c|c|c|c|}
\hline & \multicolumn{4}{|c|}{ PULPA TAMIZADA ELABORADA EN ESCALA PILOTO, ZAFRA 2010} \\
\hline & $\begin{array}{c}\text { Sólidos solubles } \\
\left({ }^{\circ} \mathrm{Brix}\right)\end{array}$ & PH & $\begin{array}{c}\text { Vitamina } C \\
\text { (mg/100gr pulpa) }\end{array}$ & $\begin{array}{l}\text { Pectina } \\
(\mathrm{g} / \mathrm{mol})\end{array}$ \\
\hline LOTE 1 & 15,3 & 3,1 & 54 & $<\mathrm{LD}$ \\
\hline LOTE 2 & 13,8 & 3,2 & 48 & $<\mathrm{LD}$ \\
\hline LOTE 3 & 13,8 & 3,3 & 40 & $<\mathrm{LD}$ \\
\hline LOTE 4 & 13,3 & 3,2 & 36 & $<\mathrm{LD}$ \\
\hline PROMEDIO & 14,0 & 3,2 & 45 & $<L D$ \\
\hline IC95\% & 1,0 & 0,1 & 9 & - \\
\hline Límite superior & 15,0 & 3,3 & 54 & $<L D$ \\
\hline Límite inferior & 13,0 & 3,1 & 35 & $<L D$ \\
\hline
\end{tabular}

Tabla 3. Caracterización de pulpa tamizada elaborada en lotes de 60 kg, zafra 2010. (LD = Límite de detección, 8E-9 g/mol)

\begin{tabular}{|c|c|c|c|c|c|c|c|c|c|c|c|}
\hline & \multicolumn{4}{|c|}{ COLOR BUTIA FRESCO } & \multicolumn{4}{|c|}{ COLOR PULPA TAMIZADA } & \multicolumn{3}{|c|}{ CAMBIOS DE COLOR } \\
\hline & Color pantone & Lo & ao & $b_{\mathbf{o}}$ & Color pantone & $\mathbf{L}_{\mathbf{o}}$ & ao & bo & $A_{\left(L^{*}-L o\right)}$ & $\mathbf{A}_{\left(\mathbf{a}^{*}-\mathbf{a o}\right)}$ & $\mathbf{A}_{\left(\mathbf{b}^{*}-\mathbf{b o}\right)}$ \\
\hline LOTE 1 & $1365 \mathrm{C}$ & 80 & 21 & 60 & $1235 \mathrm{C}$ & 79 & 22 & 85 & -1 & 1 & 25 \\
\hline LOTE 2 & $1365 \mathrm{C}$ & 80 & 21 & 60 & $1235 \mathrm{C}$ & 79 & 22 & 85 & -1 & 1 & 25 \\
\hline LOTE 3 & $1235 \mathrm{C}$ & 79 & 22 & 85 & $130 \mathrm{C}$ & 75 & 18 & 86 & -4 & -4 & 1 \\
\hline LOTE 4 & $1235 \mathrm{C}$ & 79 & 22 & 85 & $130 \mathrm{C}$ & 75 & 18 & 86 & -4 & -4 & 1 \\
\hline
\end{tabular}

Tabla 4. Medida del color por comparación Pantone en la pulpa del fruto fresco y en la pulpa tamizada.

\begin{tabular}{|c|c|c|c|c|c|c|}
\hline \multirow[b]{2}{*}{ Muestra } & \multirow{2}{*}{ Tiempo almacenamiento } & \multicolumn{5}{|c|}{ días } \\
\hline & & 0 & 90 & 180 & 270 & 360 \\
\hline \multirow{2}{*}{$\begin{array}{l}\text { Pulpa } \\
\text { tamizada } \\
\text { congelada }\end{array}$} & $\begin{array}{l}\text { Recuento de aerobios } \\
\text { (ufc/g) }\end{array}$ & 10 & $<10$ & $<10$ & 10 & 10 \\
\hline & $\begin{array}{l}\text { Recuento de hongos } \\
\text { y levaduras (ufc/g) }\end{array}$ & 30 & $<10$ & $<10$ & $<10$ & $<10$ \\
\hline
\end{tabular}

Tabla 5. Resultados microbiológicos de la pulpa tamizada congelada. 


\section{Seguimiento de la pulpa tamizada conservada por congelación}

En la Tabla 5 se describen los resultados del seguimiento microbiológico de la pulpa tamizada congelada.

Se realizó seguimiento de color de las pulpas congeladas; no se observaron cambios en todo el período de almacenamiento.

\section{Discusión}

Se destaca el alto contenido de vitamina $\mathrm{C}$ y de fibra observado en el fruto fresco, comparable al de otros frutos conocidos por estos aportes nutricionales. El intervalo de valores observados de vitamina $\mathrm{C}$ en la pulpa del fruto fresco fue de 65 a $100 \mathrm{mg}$ Vit. C/100g, comparable a los del kiwi (97.8 mg Vit.C/100 gr), frutilla (88.7 mg Vit. C/100g), limón (62.6 mg Vit. C/100g), naranja (59 mg Vit. C/100g), pomelo (48 $\mathrm{mg}$ Vit. C/100g), ananá (mg Vit. $40.3 \mathrm{C} / 100 \mathrm{~g}$ ), valores informados en Tabla de Composición de Alimentos del Uruguay (2002). El contenido de fibra total en el butiá resultó entre 4.3 y $4.9 \mathrm{~g} / 100 \mathrm{gr}$ de pulpa, comparable al contenido de fibra de limón $(4.7 \mathrm{~g} / 100 \mathrm{gr})$, naranja ( $2.3 \mathrm{~g} / 100 \mathrm{gr})$, manzana ( $2.8 \mathrm{~g} / 100 \mathrm{gr})$, según valores informados en Tabla de Composición de Alimentos del Uruguay (2002). El fruto no presentó valores detectables de pectina según el método de análisis aplicado. Los valores de sólidos solubles y acidez titulable fueron coincidentes con los reportados por Machado (2008) en pulpa de butiá del litoral medio de Rio Grande do Sul. La relación sólidos solubles/ acidez titulable fue de SST/AT=10 $\pm 2_{(\mathrm{IC}=95 \%)}$, indicando frutos con grado de madurez similares en las muestras.

La pulpa tamizada elaborada según las condiciones descriptas presentó valores de sólidos solubles y $\mathrm{pH}$ dentro del mismo rango que los frutos frescos. El contenido de vitamina $\mathrm{C}$ en la pulpa tamizada, si bien se redujo entre un $45 \%$ y $65 \%$, mantuvo valores de interés nutricional. El color de la pulpa tamizada no presentó cambios respecto al fruto fresco, ni tampoco en su almacenamiento a temperaturas de congelación, en 360 días.

El peso de las unidades de fruto fresco osciló entre $10 \mathrm{~g}$ a $8,6 \mathrm{~g}$ (IC $95 \%$ ) y el de los cocos respectivos fue de $2,5 \mathrm{~g}$ a $2,1 \mathrm{~g}$. El porcentaje de pulpa en el fruto resultó entre $69 \%$ y $61 \%$; estos valores se pueden asociar al rendimiento teórico de la elaboración de pulpa tamizada. El rendimiento del proceso de elaboración de pulpa tamizada estuvo entre el $49 \%$ y el $51 \%$. Esta pérdida de rendimiento se produjo debido a la pulpa que quedó adherida a los cocos y a la fibra del descarte del tamizado.

No se detectaron desviaciones microbiológicas durante el almacenamiento de la pulpa tamizada congelada.

\section{Conclusiones}

El fruto de la palmera Butia capitata cosechada en el departamento de Rocha en zafra 2010 presenta aportes importantes de vitamina C y de fibra dietética, comparable al de otros frutos conocidos por estos aportes nutricionales (kiwi, frutilla, limón, naranja, pomelo y ananá).

El color, contenido de sólidos solubles y $\mathrm{pH}$ del fruto fresco son similares a los de la pulpa tamizada elaborada en las condiciones descriptas. La vitamina $\mathrm{C}$ se preserva hasta en un $55 \%$ en la pulpa tamizada. Se logra un rendimiento del $50 \%$ en la producción de pulpa tamizada en relación al fruto fresco.

En las condiciones de este estudio se concluye que la pulpa de butiá tamizada puede conservarse a $-18^{\circ} \mathrm{C}$ durante 360 días para su utilización en elaboración de alimentos en base a butiá.

\section{Reconocimientos}

Agradecemos muy especialmente la colaboración de la Gerencia de Análisis y Ensayos del Laboratorio Tecnológico del Uruguay, así como a los demás integrantes del equipo del proyecto: Ing. Rivas, por el Departamento de Biología Vegetal de la Facultad de Agronomía, abocada al desarrollo de una propuesta de reglamentación de manejo extractivo de los frutos de la palmera Butiá para su conservación y utilización sustentable; Dr. Dellacassa, por la Cátedra de Farmacognosia y Productos Naturales de la Facultad de Química, en el estudio de los aromas y sabores de fruto y sus derivados; Msc. Flores en el estudio de las propiedades funcionales de fruto.

\section{Referencias}

\section{- AOAC INTERNATIONAL. Official Methods of Analysis of} AOAC International. Gaithersburg: AOAC, 1996. Official Method 985.29.

- APHA. Compendium of methods for the microbiological examination of foods. 4ta. ed. Washington: APHA, 2001a. Chapter 20-Yeasts and molds. p. 209.

- APHA. Compendium of methods for the microbiological examination of foods. 4ta. ed. Washington: APHA, 2001b. Chapter 6-Culture methods for enumeration of microorganisms. p. 53.

- APHA. Compendium of methods for the microbiological examination of foods. 4ta. ed. Washington: APHA, 2001c. Chapter 7- Aerobic plate count. p. 63.

- BETANCURT, P.; GIOSCIA, D.; AYRES, C.; ARCIA, P. Fruto autóctono butiá: innovación y transferencia tecnológica. En: INNOTEC. 2008, (3):63-71.

- BRAUSE, ALLAN R.; WOOLLARD, DAVID C.; INDYK, HARVEY E. Determination of Total vitamin $\mathrm{c}$ in fruit juices and related products by liquid chromatography: interlaboratory study. En: Journal of AOAC. 2003, 86(2):367.

- BURZACO, P.; RUIZ-DÍAZ, M. F.; SOSA, J.; PINTO, A.1. Helado. Montevideo: LATU, 2010a. (Serie Frutos Nativos: Butiá; 1).

- BURZACO, P.; RUIZ-DÍAZ, M. F.; SOSA, J.; PINTO, A. Salsa para postres y helados. Montevideo: LATU, 2010b. (Serie Frutos Nativos: Butiá; 2).

- BURZACO, P.; RUIZ-DÍAZ, M. F.; SOSA, J.; PINTO, A. Mermelada y dulce de corte. Montevideo: LATU, 2010c. (Serie Frutos Nativos: Butiá; 3).

- INTERNATIONAL STANDARD ORGANIZATION (Switzerland). ISO 750: Fruit and vegetable products -Determination of titratable acidity. Geneva: ISO, 1998.

- KIRK, R.; SAWYER, R. Pearson's composition and analysis of foods. London: Longman, 1991.

- MACHADO, S., TONIETTO, A., SCHLINDWEIN, G., DUARTE, A. C., DUPRAT, Á. COSTA, R. Caracterização química da polpa de Butiá (Butia Capitata Mart.) procedente do litoral médio do Rio Grande do Sul. En: SOCIEDADE BRASILEIRA DE FRUTICULTURA. XX Congresso Brasileiro de Fruticultura, 54th Annual Meeting of the Interamerican Society for Tropical Horticulture (Brasil 12-17 de octrubre de 2008). [s.1.]: SBF, 2008.

- RIVAS, M.; BARILANI, A. Diversidad, potencial productivo y reproductivo de los palmares de Butia capitata (Mart.) Becc. de Uruguay. En: Agrociencia. 2004, VII(1):11-20. 